\title{
Blanc-Chaleard Marie-Claude, et alii (textes réunis et présentés par), Police et migrants, France 1667-1939
}

Jean-François Tanguy

\section{OpenEdition}

1 Journals

\section{Édition électronique}

URL : http://journals.openedition.org/abpo/1651

DOI : 10.4000/abpo.1651

ISBN : 978-2-7535-1485-0

ISSN : 2108-6443

\section{Éditeur}

Presses universitaires de Rennes

Édition imprimée

Date de publication : 20 mars 2002

Pagination : $176-178$

ISBN : 978-2-86847-708-8

ISSN : 0399-0826

\section{Référence électronique}

Jean-François Tanguy, «Blanc-Chaleard Marie-Claude, et alii (textes réunis et présentés par), Police et migrants, France 1667-1939», Annales de Bretagne et des Pays de l'Ouest [En ligne], 109-1 | 2002, mis en ligne le 20 mars 2004, consulté le 23 septembre 2020. URL : http://journals.openedition.org/abpo/ 1651 ; DOI : https://doi.org/10.4000/abpo.1651 
fère l'église paroissiale à l'Hôtel-Dieu, fait retraite plus que charité, lit surtout des œuvres spirituelles où la dévotion se veut intérieure et discrète. Et La Bruyère raille volontiers la mondanité du "sermon de charité ", comme si la rhétorique sacrée était devenue l'ultime refuge du "formidable élan de solidarité " - comme on dit aujourd'hui - des décennies précédentes.

On mesure à ce bref résumé la richesse d'un ouvrage à la progression convaincante, où de fermes conclusions viennent résumer à propos une démonstration parfois tortueuse dans le détail de son argumentation. Certaines imperfections formelles, notamment dans le renvoi aux annexes, n'affectent pas l'intérêt de ce qui est sans nul doute une étude de référence sur la "sensibilité charitable ". De grande importance pour l'histoire de Paris, ce livre dépasse évidemment aussi l'horizon de la capitale : d'abord parce que celle-ci est un révélateur privilégié d'évolutions plus générales, mais aussi parce que la littérature de spiritualité analysée par l'auteur n'est parisienne que par son lieu d'édition; elle a en réalité connu une audience bien plus large. On se gardera pourtant d'ériger le cas parisien en modèle unique, ce que l'auteur n'entend nullement faire : la Bretagne, bien connue par les travaux d'A. Croix, J.-L. Bruzulier ou G. Haudebourg, est là pour rappeler la force de l'attachement à «l'aumône manuelle " et sa durable résistance à la " charité institutionnelle ".

Georges Provost

Blanc-Chaleard, Marie-Claude, Douli, Caroline, Dyonet, Nicole et Milliot, Vincent (textes réunis et présentés par), Police et migrants, France 1667-1939, actes du colloque " Police et migrants, France 1667-1945 " organisé à l'université d'Orléans les 28 et 29 octobre 1999, Rennes, PUR, 2001.

Le colloque organisé à l'université d'Orléans en octobre 1999 posait une question relativement nouvelle mais perspicace : la police, au sens contemporain du terme, pousse-t-elle une partie de ses racines historiques dans le contrôle, non des populations résidentes, mais dans celui des migrants, des étrangers, des nomades, supposés représenter l'inconnu, I'inhabituel et donc le danger?

Les actes ici rassemblés pourraient incliner à répondre oui : "La mobilité apparaît comme l'un des moteurs de la réflexion sur la police générale et particulière et sur l'inflexion de ses démarches qui entrainent les comportements de tous " (Daniel Roche, Introduction, p. 31). Certes, on a par ailleurs la preuve que la police possède aussi d'autres origines : espionnage et renseignement politique, protection des puissants, lutte contre les opposants, maintien des fonctions de base de la cité. Reste que la démonstration est ici bien faite qu'une des voies de développement de la police a reposé sur le contrôle, la mise à l'écart ou l'expulsion des corps étrangers. La frontière entre ces différentes catégories d'activités reste par ailleurs floue et perméable.

Un certain nombre de constantes apparaissent dans des articles pourtant divers, même si l'évolution des mœurs et des techniques peut infléchir la forme de ces invariants :

- Les buts avoués du contrôle : le migrant est toujours vu comme un élément hors des normes dans un État policé (aux deux sens du terme justement). Le bon sujet (ou citoyen) ne se déplace pas. Partir est toujours la manifestation d'une insatisfaction ou de pulsions dangereuses. "On retrouve toujours, dans les représentations, les mêmes soupçons attachés aux errants et nomades : la concur- 
rence aux activités régulières et patentées, les risques encourus au plan moral (délinquance) et sanitaire (le nomade vecteur de maladie) ${ }^{4}$ " (Jean-Claude Farcy, "Le Contrôle des migrants en ville ", Introduction, p. 282). Tout étranger - au sens large - est un fauteur de troubles potentiel, membre de la cohorte des " opposants politiques [des] marginaux divers [des] ouvriers coalisés [des] insoumis et [des] déserteurs enfin qu'il convient de mettre au pas ", programme prêté à Fouché (Vincent Denis, " Le contrôle de la mobilité à travers les passeports sous l'Empire ", p. 76), mais que d'innombrables responsables de l'Ordre auraient pu reprendre au fil des temps. Il y a dans a ordre " un forte connotation de "disposition régulière, rationnelle ». Le migrant n'est ni organisé, ni prévisible.

- Les techniques du contrôle : dès le XvII siècle, le recensement et la surveillance des migrants repose sur la dévolution de fonctions adéquates à un ou plusieurs corps spécialisés, ce qui ne veut pas dire ipso facto professionnels, « la surveillance des étrangers étant un des devoirs des membres de la commune bourgeoise " (Catherine Clémens-Denys, " Les transformations du contrôle des étrangers dans les villes de la frontière du Nord, 1667-1789 ", p. 211). À Toulouse, sous l'Ancien Régime, le contrôle des étrangers repose en partie sur les dizeniers, "particuliers nommés par les capitouls à la tête du moulon (ou de la dizaine) où ils résidaient " (Jean-Luc Laffont, "La police des étrangers à Toulouse sous l'Ancien Régime ", p. 296). Bien entendu, plus le temps passera et plus les fonctions de contrôle tomberont aux mains d'une police professionnelle. Reste que les particuliers seront toujours censés participer à de telles détections, identifications et précautions, que ce soit ès qualités comme les patrons de " garnis " (Claire Levy-Vroelant, " Un siècle de surveillance des garnis à Versailles, 1830-1930") ou les logeurs de tout poil (Vincent Milliot, « Migrants et " étrangers ", sous l'œil de la police : la surveillance des lieux d'accueil parisiens au siècle des Lumières "), ou même les simples citoyens, notamment en temps de guerre (Philippe Rygiel signale des rondes de "propriétaires " dans le Berry de la Grande guerre aux fins d'appréhender des étrangers maraudeurs - " Police, étrangers et travailleurs coloniaux dans le Cher de 1914 à 1918 "). Les autres techniques permanentes de contrôle font très tôt appel aux papiers et registres, notamment de logeurs, puis aux passeports systématisés mais non inventés par la Révolution et l'Empire, livrets ouvriers, documents en tous genres.

On ne doit pas uniquement - et l'ouvrage s'en garde bien - scruter les constantes. On devra aussi admettre que les méthodes (les méthodes beaucoup plus que les fins...) évoluent en fonction des avancées techniques ou des mutations de la société. Le plus important est certainement le développement progressif des forces de police, de plus en plus spécialisées. De ce point de vue, on distinguera une césure méthodologique, celle qui voit la création par Fouché d'une police effficace sur un plan national, et une césure effective, celle qui s'incarne pendant la seconde moitié du XIX ${ }^{\mathrm{e}}$ siècle dans la montée en puissance des effectifs policiers, puis des techniques afférentes. Peut-être pourra-t-on regretter quelque peu que cet aspect essentiel (la modernisation des méthodes) soit surtout abordé dans la postface de Jean- Marc Berlière et René Lévy (p. 395 et suivantes). Le rôle des commissaires spéciaux, des brigades mobiles, des techniques de bertillonnage remplissent à l'évidence une fonction majeure dans le contrôle des migrants depuis les premières annces du $\mathrm{Xx}^{\mathrm{e}}$ siècle au moins.

Mais par ailleurs plusieurs articles, sinon tous, soulignent les difficultés à remplir le programme établi et les innombrables dysfonctionnements qui limi-

4. On remarquera - sans y attacher une importance excessive - que l'équivalent grec du mot d'origine latine migration est métastase (= déplacement), qui ne s'applique en fiançais moderne qu'au cancer. Est-ce un hasard? 
tent l'effficacité du contrôle. En fait, les autorités veulent tout savoir, ou en tout cas elles prétendent le vouloir, mais dans la réalité elles en savent peu, et même beaucoup moins qu'elles ne le prétendent. Les raisons de cet état de fait sont multiples : insufffisance chronique des effectifs (Philippe Rygiel, Claire LevyVroelant), médiocrité des personnels concernés (Philippe Rygiel, Vincent Milliot), intérêts divers, et notamment économiques, des populations ou même simplement d'une partie d'entre elles à ne pas donner aux autorités toutes les indications nécessaires (Gérard Le Bouëdec, « Police et migrants d'une ville portuaire : Lorient au XVIII ${ }^{\mathrm{e}}$ siècle »; Émile Temime, «Immigration et police portuaire à la fin du XIX ${ }^{\mathrm{e}}$ siècle : le cas de Marseille "; Claire Zalc, "Contrôler et surveiller le commerce migrant. Nomades, forains et ambulants à Paris [1912-1940] ", etc.); solidarité active (et parfois des policiers eux-mêmes!) envers certaines catégories de migrants, soit par sentiment politique (Ralph Schor, « La police et la surveillance de la frontière des Alpes-Maritimes de 1919 à 1939 "), soit par solidarité de proximité ou de classe comme l'on voudra : une catégorie particulière de migrants demeure au moins jusqu'à la fin du XIXe siècle protégée et aidée par les populations locales, les déserteurs (Arnaud-Dominique Houté, « Le migrant du gendarme. Le quotidien de la surveillance dans le département du Nord pendant la première moitié du xIXe siècle ").

Enfin, on peut se demander si parfois le contrôle des migrants se donne vraiment pour but ses objectifs apparents ou si, comme le souligne C. Clémens- Denys, il ne s'agit pas en partie d'un " laboratoire " destiné à rôder et à perfectionner des pratiques applicables ensuite à l'ensemble de la population; ou même si l'activité en question n'est pas destinée occasionnellement à justifier le travail et l'existence des forces de l'ordre elle-mêmes, tant la répression des migrants apparaît comme un travail simple dans ses modalités - sinon dans la pratique - et clairement identifiable (A.-D. Houté), bref une occupation surtout destinée à " faire du chiffre " comme l'on dirait aujourd'hui. En partie sans doute, même si on ne doit pas tomber d'un excès dans l'autre, et supposer que les pouvoirs publics ont moins pour but d'embrasser d'un regard panoptique toute la société que de lui prouver qu'ils servent à quelque chose, ce dont de temps à autre on pourrait douter.

Au total, on n'a épuisé ici qu'une partie des riches informations et problématiques développées dans ce volume. On ne déplorera pas qu'une partie notable des migrants (vagabonds, bohémiens, commerçants, touristes mêmes) soit absente ou peu présente " de face " ici. Il était question de la façon dont la police et les autorités de police traitent les migrations, non des migrants eux- mêmes. Peut-être regrettera-t-on davantage que la répartition (le hasard?) des communications ait privilégié le milieu urbain au détriment des campagnes comme si celles-ci étaient (faussement à notre avis) moins concernées (outre A.-D. Houté, déjà cité, signalons Nicole Dyonet, "La maréchaussée et la population mobile dans l'Orléanais au XVIII ${ }^{e}$ siècle ")? Ou que ne coure qu'en filigrane l'itérative question : les moyens et le nombre des forces de l'ordre a prodigieusement augmenté depuis trois siècles et demi, mais le volume des migrants aussi; le contrôle est-il donc plus, moins ou ni plus ni moins effficace qu'en 1667? Énigme insoluble? Aux dernières lignes du volume, J.-M. Berlière et R. Lévy évoquent des " sociétés de masse contemporaines [...] beaucoup moins contrôlées que les micro-sociétés d'antan soumises au contrôle communautaire et aux regards croisés du voisinage " (p. 415). C'est nous semble-t-il supposer résolu, de manière fort optimiste, un problème dont les termes ne sont encore que très imparfaitement ou en tout cas très empiriquement définis. 